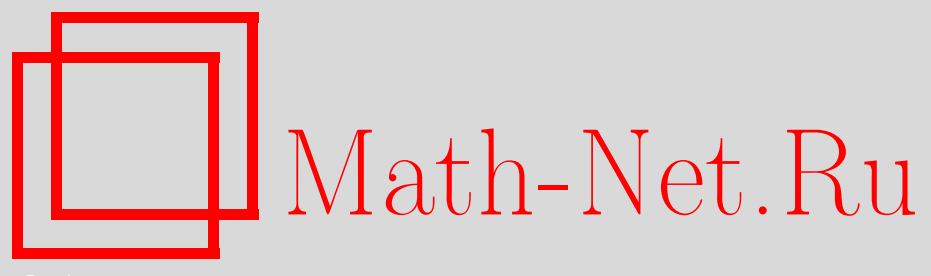

Р. М. Хакимов, Единственность слабо периодической гиббсовской меры для НС-модели, Матем. заметки, 2013, том 94, выпуск 5, 796-800

DOI: https://doi.org/10.4213/mzm9687

Использование Общероссийского математического портала Math-Net.Ru подразумевает, что вы прочитали и согласны с пользовательским соглашением http://www . mathnet.ru/rus/agreement

Параметры загрузки:

IP : 3.85 .73 .92

26 апреля 2023 г., 06:27:28

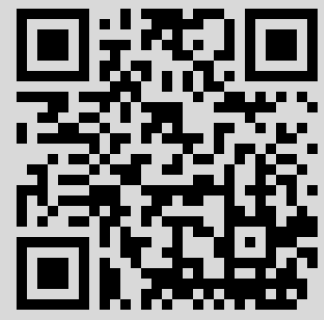




\section{Единственность слабо периодической гиббсовской меры для НС-модели}

\section{Р. М. Хакимов}

Пусть $\tau^{k}=(V, L)$ - дерево Кэли порядка $k \geqslant 1$, т.е. бесконечное дерево, из каждой вершины которого выходит ровно $k+1$ ребер, где $V, L$ - есть множество вершин и ребер дерева $\tau^{k}$, соответственно.

Для любого фиксированного $x^{0} \in V$ обозначим

$$
W_{n}=\left\{x \in V \mid d\left(x, x^{0}\right)=n\right\}, \quad V_{n}=\left\{x \in V \mid d\left(x, x^{0}\right) \leqslant n\right\},
$$

где $d(x, y)$ - расстояние между $x, y$ на дереве Кэли, т.е. число ребер пути, соединяющего $x$ и $y$.

Известно, что дерево Кэли представляется как группа $G_{k}$, являющаяся свободным произведением $k+1$ циклических групп второго порядка с образующими $a_{1}, \ldots, a_{k+1}$. Пусть $\Phi=\{0,1\}$ и $\sigma \in \Phi^{V}$ - конфигурация, т.е. $\sigma=\{\sigma(x) \in \Phi: x \in V\}$, где $\sigma(x)=1$ означает, что вершина $x$ на дереве Кэли занятая, а $\sigma(x)=0$ означает, что она свободная. Конфигурация $\sigma$ называется допустимой, если $\sigma(x) \sigma(y)=0$ для любых соседних $\langle x, y\rangle$ из $V$ $\left(V_{n}\right.$ или $W_{n}$, соответственно); обозначим множество таких конфигураций через $\Omega\left(\Omega_{V_{n}}\right.$ и $\left.\Omega_{W_{n}}\right)$. Ясно, что $\Omega \subset \Phi^{V}$.

Гамильтониан НC (Hard Core)-модели определяется по формуле

$$
H(\sigma)= \begin{cases}J \sum_{x \in V} \sigma(x), & \text { если } \sigma \in \Omega, \\ +\infty, & \text { если } \sigma \notin \Omega,\end{cases}
$$

где $J \in R$.

Основной задачей является изучение предельных гиббсовских мер. В общем случае существование предельных гиббсовских мер для достаточно широкого класса гамильтонианов доказана Добрушиным (см. например [1]). Известно [2], что каждой мере Гиббса для НС-модели на дереве Кэли можно сопоставить совокупность величин $z=\left\{z_{x}, x \in G_{k}\right\}$, удовлетворяющих

$$
z_{x}=\prod_{y \in S(x)}\left(1+\lambda z_{y}\right)^{-1}
$$

где $\lambda=e^{J}>0$ - параметр, $S(x)$ - множество "прямых потомков" точки $x \in V$. Кроме того, для $x \in V$ показана следующая оценка (см. [2]):

$$
(1+\lambda)^{-k}<z_{x}<1 .
$$

Пусть $G_{k} / \widehat{G}_{k}=\left\{H_{1}, \ldots, H_{r}\right\}-$ фактор группа, где $\widehat{G}_{k}$ - нормальный делитель индекса $r \geqslant 1$

Если гиббсовская мера инвариантна относительно некоторой подгруппы конечного индекса $\widehat{G}_{k} \subset G_{k}$, то она называется $\widehat{G}_{k}$-периодической. $G_{k}$-периодические совокупности называются трансляиионно-инвариантными [1], [3].

В многочисленных работах (см. например [2], [4], [5]) на дереве Кэли изучены периодические меры Гиббса для различных моделей статистической механики. Эти меры, в основном, были трансляционно-инвариантными либо периодическими с периодом два. В работе [6] введены более общие понятия периодической меры Гиббса, т.е. слабо периодические гиббсовские меры, и доказано существование таких мер для модели Изинга на дереве Кэли. В работе [2] изучены Гиббсовские меры для НС-модели на дереве Кэли и доказано, что трансляционно-инвариантная мера Гиббса для этой модели единственна. Кроме того, при

DOI: $10.4213 / \operatorname{mzm} 9687$ 
некоторых условиях на параметры НС-модели доказана неединственность периодических мер Гиббса с периодом два.

Эта работа посвящена изучению слабо периодических мер Гиббса для НС-модели на дереве Кэли для любого нормального делителя индекса два и доказывается единственность таких мер.

ОПределЕние 1 . Совокупность величин $z=\left\{z_{x}, x \in G_{k}\right\}$ называется $\widehat{G}_{k}$-периодической, если $z_{y x}=z_{x}$ для всех $x \in G_{k}, y \in \widehat{G}_{k}$. $G_{k}$-периодические совокупности называются транслячионно-инвариантными.

Для любого $x \in G_{k}$ положим $x_{\downarrow}=\left\{y \in G_{k}:\langle x, y\rangle\right\} \backslash S(x)$ (см. [6]).

ОПредЕлЕние 2. Совокупность величин $z=\left\{z_{x}, x \in G_{k}\right\}$ называется $\widehat{G}_{k}$-слабо периодической, если $z_{x}=z_{i j}$ при $x \in H_{i}, x_{\downarrow} \in H_{j}$ для всех $x \in G_{k}$.

ОПРедЕЛЕниЕ 3 . Мера $\mu$ называется $\widehat{G}_{k}$-(слабо) периодической, если она соответствует $\widehat{G}_{k^{-}}$(слабо) периодической совокупности величин $z$.

Пусть $\varnothing \neq A \subset N_{k}=\{1,2, \ldots, k+1\}$. Известно, что любая подгруппа индекса два группы $G_{k}$ имеет вид

$$
H_{A}=\left\{x \in G_{k}: \sum_{i \in A} w_{x}\left(a_{i}\right) \text { - четное число }\right\},
$$

где $w_{x}\left(a_{i}\right)$ - число букв $a_{i}$ в слове $x \in G_{k}$ (см. [4]).

Заметим, что $H_{A}$-слабо периодическая совокупность $z$ имеет вид

$$
z_{x}= \begin{cases}z_{1}, & x \in H_{A}, x_{\downarrow} \in H_{A}, \\ z_{2}, & x \in H_{A}, x_{\downarrow} \in G_{k} \backslash H_{A}, \\ z_{3}, & x \in G_{k} \backslash H_{A}, x_{\downarrow} \in H_{A}, \\ z_{4}, & x \in G_{k} \backslash H_{A}, x_{\downarrow} \in G_{k} \backslash H_{A} .\end{cases}
$$

Тогда в силу (1) $z_{i}, i=1, \ldots, 4$, удовлетворяют следующей системе уравнений:

$$
\begin{array}{rlrl}
z_{1} & =\left(\frac{1+\lambda z_{1}}{1+\lambda z_{3}}\right)^{i} \cdot \frac{1}{\left(1+\lambda z_{1}\right)^{k}}, & z_{2} & =\left(\frac{1+\lambda z_{1}}{1+\lambda z_{3}}\right)^{i-1} \cdot \frac{1}{\left(1+\lambda z_{1}\right)^{k}}, \\
z_{3}=\left(\frac{1+\lambda z_{4}}{1+\lambda z_{2}}\right)^{i-1} \cdot \frac{1}{\left(1+\lambda z_{4}\right)^{k}}, & z_{4}=\left(\frac{1+\lambda z_{4}}{1+\lambda z_{2}}\right)^{i} \cdot \frac{1}{\left(1+\lambda z_{4}\right)^{k}},
\end{array}
$$

где $|A|=i$; здесь $|A|$ обозначает число элементов множества $A$. Разделив в этой системе уравнений первое уравнение на второе, третье на четвертое, получим следующую систему уравнений:

$$
\frac{z_{1}}{z_{2}}=\frac{1+\lambda z_{1}}{1+\lambda z_{3}}, \quad \frac{z_{3}}{z_{4}}=\frac{1+\lambda z_{2}}{1+\lambda z_{4}} .
$$

Используя эту систему уравнений, (3) можно переписать как

$$
\begin{aligned}
z_{1} & =\left(\frac{z_{1}}{z_{2}}\right)^{i} \cdot \frac{1}{\left(1+\lambda z_{1}\right)^{k}}, & z_{2} & =\left(\frac{z_{1}}{z_{2}}\right)^{i-1} \cdot \frac{1}{\left(1+\lambda z_{1}\right)^{k}}, \\
z_{3} & =\left(\frac{z_{4}}{z_{3}}\right)^{i-1} \cdot \frac{1}{\left(1+\lambda z_{4}\right)^{k}}, & z_{4} & =\left(\frac{z_{4}}{z_{3}}\right)^{i} \cdot \frac{1}{\left(1+\lambda z_{4}\right)^{k}} .
\end{aligned}
$$

Из первого уравнения системы (5) найдем $z_{2}$, из четвертого $z_{3}$ и, подставив их в четвертое и первое уравнения системы (3), соответственно, получим

$$
\begin{aligned}
& z_{1}=\frac{\left(1+\lambda z_{4}\right)^{k}}{\left(\left(1+\lambda z_{4}\right)^{k / i}+\lambda z_{4}^{1-1 / i}\right)^{i}} \cdot \frac{1}{\left(1+\lambda z_{1}\right)^{k-i}}, \\
& z_{4}=\frac{\left(1+\lambda z_{1}\right)^{k}}{\left(\left(1+\lambda z_{1}\right)^{k / i}+\lambda z_{1}^{1-1 / i}\right)^{i}} \cdot \frac{1}{\left(1+\lambda z_{4}\right)^{k-i}}
\end{aligned}
$$


или

$$
z_{1}=\varphi\left(z_{4}\right) \cdot \psi\left(z_{1}\right), \quad z_{4}=\varphi\left(z_{1}\right) \cdot \psi\left(z_{4}\right),
$$

где

$$
\varphi(z)=\frac{(1+\lambda z)^{k}}{\left((1+\lambda z)^{k / i}+\lambda z^{1-1 / i}\right)^{i}}, \quad \psi(z)=\frac{1}{(1+\lambda z)^{k-i}} .
$$

Следующая лемма полезна для наших исследований.

Лемма. 1) Если в системе уравнений (3) выполнены равенства $z_{1}=z_{4}, z_{2}=z_{3}$, то $z_{1}=z_{2}=z_{3}=z_{4}$.

2) Тогда и только тогда $z_{1}=z_{4}$, когда $z_{2}=z_{3}$.

Доказательство. 1) Пусть $z_{2}=z_{3}$; тогда из первого уравнения (4) имеем $z_{1} / z_{2}=$ $\left(1+\lambda z_{1}\right) /\left(1+\lambda z_{2}\right)$, откуда $z_{1}=z_{2}$. Кроме того, по условию леммы $z_{1}=z_{4} ;$ следовательно, $z_{1}=z_{2}=z_{3}=z_{4}$.

2) Пусть $z_{1}=z_{4}$; тогда из (4) имеем $z_{3} / z_{2}=\left(1+\lambda z_{2}\right) /\left(1+\lambda z_{3}\right)$, откуда $z_{2}=z_{3}$. Пусть $z_{2}=z_{3}$; тогда из (4) имеем $z_{1} / z_{4}=\left(1+\lambda z_{1}\right) /\left(1+\lambda z_{4}\right)$, откуда получим $z_{1}=z_{4}$. Лемма доказана.

Теорема. Для любых $k \geqslant 1, i \leqslant k$, где $i=|A|$, и при любом $\lambda>0$ для $H C$-модели $H_{A}$-слабо периодическая мера Гиббса единственна. Более того, эта мера совпадает с единственной трансляиионно-инвариантной мерой Гиббса.

ДокАзАтельство. Для доказательства достаточно показать, что система функциональных уравнений (6) имеет корни только вида $z_{1}=z_{2}=z_{3}=z_{4}$. Для этого в (6) вычтем из первого уравнения второе и в правую часть полученного уравнения прибавим и вычтем $\varphi\left(z_{1}\right) \cdot \psi\left(z_{1}\right)$, затем используя теорему Лагранжа о среднем для функций $\varphi(z)$ и $\psi(z)$ на $\left(z_{1}, z_{4}\right)$, получим

$$
z_{1}-z_{4}=\psi\left(z_{1}\right) \cdot \varphi^{\prime}(\xi) \cdot\left(z_{4}-z_{1}\right)+\varphi\left(z_{1}\right) \cdot \psi^{\prime}(\eta) \cdot\left(z_{1}-z_{4}\right),
$$

где $\xi \in\left(z_{1}, z_{4}\right), \eta \in\left(z_{1}, z_{4}\right)$. Отсюда

$$
\left(z_{1}-z_{4}\right) \cdot\left[1+\psi\left(z_{1}\right) \cdot \varphi^{\prime}(\xi)-\varphi\left(z_{1}\right) \cdot \psi^{\prime}(\eta)\right]=0 .
$$

Из этого уравнения получим, что $z_{1}=z_{4}$ или

$$
1+F\left(z_{1}, \xi, \eta\right)=0
$$

где

$$
F\left(z_{1}, \xi, \eta\right)=\psi\left(z_{1}\right) \cdot \varphi^{\prime}(\xi)-\varphi\left(z_{1}\right) \cdot \psi^{\prime}(\eta)
$$

Здесь $\psi^{\prime}(\eta)=-\lambda(k-i) /(1+\lambda \eta)^{k-i+1} \leqslant 0$, т.е. функция $\psi(\eta)$ убывает, так как $i \leqslant k, \lambda>0$, $\eta>0$, а производная функции $\varphi(z)$ в точке $\xi \in\left(z_{1}, z_{4}\right)$ имеет вид

$$
\varphi^{\prime}(\xi)=\frac{\lambda(1+\lambda \xi)^{k-1}[\lambda \xi(k-i+1)-i+1]}{\xi^{1 / i}\left[(1+\lambda \xi)^{k / i}+\lambda \xi^{1-1 / i}\right]^{i+1}} .
$$

Всевозможные значения $(i, k)$ разделим на два случая.

1) Случай $i \leqslant(k+1) / 2$. Покажем, что уравнение (8) не имеет решений, т.е.

$$
F\left(z_{1}, \xi, \eta\right)>-1 .
$$

Для этого, используя (2), рассмотрим следующую оценку:

$$
\begin{gathered}
F\left(z_{1}, \xi, \eta\right)>\frac{\lambda\left(1+\lambda /(1+\lambda)^{k}\right)^{k-1}\left[\lambda(k-i+1) /(1+\lambda)^{k}-i+1\right]}{(1+\lambda)^{k-i}\left[(1+\lambda)^{k / i}+\lambda\right]^{i+1}} \\
+\frac{\lambda(k-i)\left(1+\lambda /(1+\lambda)^{k}\right)^{k}}{\left[(1+\lambda)^{k / i}+\lambda\right]^{i}(1+\lambda)^{k-i+1}}
\end{gathered}
$$




$$
\begin{aligned}
& >\frac{\lambda\left((1+\lambda)^{k}+\lambda\right)^{k-1}\left[\lambda(k-i+1)+(1-i)(1+\lambda)^{k}\right]}{(1+\lambda)^{k^{2}+k-i+1}\left[(1+\lambda)^{k}+\lambda\right]^{i+1}} \\
& \quad+\frac{\lambda(k-i)\left((1+\lambda)^{k}+\lambda\right)^{k}}{(1+\lambda)^{k^{2}+k-i+1}\left[(1+\lambda)^{k}+\lambda\right]^{i}} \\
& >-1 .
\end{aligned}
$$

Отсюда

$$
\begin{aligned}
& \lambda\left((1+\lambda)^{k}+\lambda\right)^{k-i-2}\left[\lambda(k-i+1)+(1-i)(1+\lambda)^{k}\right] \\
& \quad+\lambda(k-i)\left((1+\lambda)^{k}+\lambda\right)^{k-i}+(1+\lambda)^{k^{2}+k-i+1}>0 .
\end{aligned}
$$

Введя обозначение $a=1+\lambda$, перепишем последнее неравенство:

$$
\lambda\left(a^{k}+\lambda\right)^{k-i-2}\left[\lambda(k-i+1)+(1-i) a^{k}\right]+\lambda(k-i)\left(a^{k}+\lambda\right)^{k-i}+a^{k^{2}+k-i+1}>0
$$

или

$$
\lambda\left(a^{k}+\lambda\right)^{k-i}\left[\lambda(k-i+1)+(k-i)\left(a^{k}+\lambda\right)^{2}-(i-1) a^{k}\right]+a^{k^{2}+k-i+1}\left(a^{k}+\lambda\right)^{2}>0 .
$$

Покажем, что разность $(k-i)\left(a^{k}+\lambda\right)^{2}-(i-1) a^{k}$ положительна. Действительно,

$$
(k-i)\left(a^{k}+\lambda\right)^{2}-(i-1) a^{k}=a^{k}\left[(k-i) a^{k}-(i-1)\right]+2(k-i) \lambda a^{k}+(k-i) \lambda^{2} .
$$

Учитывая то, что $a=1+\lambda$, рассмотрим разность

$$
(k-i) a^{k}-(i-1)=(k-i)(1+\lambda)^{k}-(i-1)=k-i+(k-i) k \lambda+\cdots+(k-i) \lambda^{k}-(i-1) .
$$

Отсюда ясно, что последнее выражение положительно, если $k-2 i+1 \geqslant 0$ или, что то же самое, $i \leqslant(k+1) / 2$. Следовательно, $\varphi^{\prime}(\xi)>-1$, а это значит, что уравнение (7) при $i \leqslant(k+1) / 2$ имеет корни только вида $z_{1}=z_{4}$; следовательно, по выше доказанной лемме система уравнений (3) при этом условии имеет единственное решение вида $z_{1}=z_{2}=$ $z_{3}=z_{4}$.

2) Случай $(k+1) / 2<i \leqslant k$. При $i=k$ доказательство очевидно. Рассмотрим $i$, $(k+1) / 2<i \leqslant k-1$. В этом случае тоже достаточно доказать неравенство (9). Аналогично предыдущему, используя (2), можно получить следующую оценку:

$$
\begin{aligned}
\psi\left(z_{1}\right) \cdot \varphi^{\prime}(\xi)-\varphi\left(z_{1}\right) \cdot \psi^{\prime}(\eta)> & \frac{\lambda\left[(1+\lambda)^{k}+\lambda\right]^{k-1} \cdot\left[\lambda(k+1-i)-(i-1)(1+\lambda)^{k}\right]}{(1+\lambda)^{k^{2}+k-i}\left[(1+\lambda)^{k / i}+\lambda\right]^{i+1}} \\
& +\frac{\lambda(k-i)\left[(1+\lambda)^{k}+\lambda\right]^{k}}{(1+\lambda)^{k^{2}+k-i+1}\left[(1+\lambda)^{k / i}+\lambda\right]^{i}} .
\end{aligned}
$$

Используя условие 2) и при этом уменьшая числитель и увеличивая знаменатель каждой дроби, перепишем эту оценку:

$$
\begin{aligned}
\psi\left(z_{1}\right) \cdot & \varphi^{\prime}(\xi)-\varphi\left(z_{1}\right) \cdot \psi^{\prime}(\eta) \\
> & \frac{\lambda\left[(1+\lambda)^{k}+\lambda\right]^{k-1} \cdot\left[\lambda(k+1-(k-1))-(k-1-1)(1+\lambda)^{k}\right]}{(1+\lambda)^{k^{2}+k-(k+1) / 2} \cdot\left[(1+\lambda)^{k /((k+1) / 2)}+\lambda\right]^{k-1+1}} \\
& \quad+\frac{\lambda(k-(k-1)) \cdot\left[(1+\lambda)^{k}+\lambda\right]^{k}}{(1+\lambda)^{k^{2}+k-(k+1) / 2+1} \cdot\left[(1+\lambda)^{k /((k+1) / 2)}+\lambda\right]^{k-1}} \\
> & \frac{\lambda\left[(1+\lambda)^{k}+\lambda\right]^{k-1} \cdot\left[2 \lambda-(k-2)(1+\lambda)^{k}\right]}{(1+\lambda)^{2 k^{2}+k+1} \cdot\left[(1+\lambda)^{k}+\lambda\right]^{k}}+\frac{\lambda \cdot\left[(1+\lambda)^{k}+\lambda\right]^{k}}{(1+\lambda)^{2 k^{2}+k+1} \cdot\left[(1+\lambda)^{k}+\lambda\right]^{k}} \\
= & \frac{\lambda \cdot\left[2 \lambda-(k-2)(1+\lambda)^{k}\right]}{(1+\lambda)^{2 k^{2}+k+1} \cdot\left[(1+\lambda)^{k}+\lambda\right]}+\frac{\lambda}{(1+\lambda)^{2 k^{2}+k+1}}>-1 .
\end{aligned}
$$


Отсюда

$$
\begin{gathered}
\lambda \cdot\left[2 \lambda-(k-2)(1+\lambda)^{k}\right]+\lambda \cdot\left[(1+\lambda)^{k}+\lambda\right]+(1+\lambda)^{2 k^{2}+k+1} \cdot\left[(1+\lambda)^{k}+\lambda\right] \\
=3 \lambda^{2}+(1+\lambda)^{k} \cdot\left[\lambda+\lambda(1+\lambda)^{2 k^{2}+1}+(1+\lambda)^{2 k^{2}+k+1}-(k-2) \lambda\right]>0 .
\end{gathered}
$$

Последнее неравенство верно, так как разность $(1+\lambda)^{2 k^{2}+k+1}-(k-2) \lambda$ положительна. Теорема доказана.

\section{СПИСОК ЦИТИРОВАННОЙ ЛИТЕРАТУРЫ}

[1] Х.-О. Георги, Гиббсовские меры и фазовые переходы, Мир, М., 1992. [2] Y. Suhov, U. A. Rozikov, Queueing Syst., 46:1-2 (2004), 197-212. [3] Я. Г. Синай, Теория фбазовых переходов. Строгие результатьи, Наука, М., 1980. [4] Н. Н. Ганиходжаев, У. А. Розиков, ТМФ, 111:1 (1997), 109-117. [5] У. А. Розиков, ТМФ, 112:1 (1997), 170-175. [6] У. А. Розиков, М. М. Рахматуллаев, ТMФ, 160:3 (2009), 507-516. 